\title{
FLUSHING EM VACAS DE CORTE NO PÓS-PARTO, SUBMETIDAS AO DESMAME PRECOCE: DESEMPENHO REPRODUTIVO
}

\author{
FLUSHING ON THE POSTPARTUM BEEF COWS SUBMITTED TO EARLY WEANING: \\ REPRODUCTIVEPERFORMANCE
}

\author{
Muller, M. ${ }^{1}$, Prado, I.N. ${ }^{2}$, Zeoula, L.M. ${ }^{2}$, Perotto, D. ${ }^{3}$, Moletta, J.L. ${ }^{3}$ e Silva, R.R. ${ }^{4}$
}

\begin{abstract}
${ }^{1}$ Pontifícia Universidade Católica do Paraná. PUCPR. Campus Toledo. Av. da União, 500. CEP 85902-532 Toledo. PR. Brasil. mylene@rla01.pucpr.br

2Universidade Estadual de Maringá (UEM). Brasil. inprado@uem.br

3IAPAR. Rodovia Celso Garcia Cid, km 375. CEP 86047-902. Três Marcos. Caixa Postal 481. Brasil. ${ }^{4}$ Universidade Estadual do Sudoeste da Bahia (UESB). Pça Primavera, 40. Bairro Primavera. CEP 45700000. Itapetinga-BA. Brasil. rrsilva.uesb@hotmail.com
\end{abstract}

\section{PalaVRas ChaVE ADICIONAIS}

Anestro. Estro. Intervalo entre partos. Lipídios.

\section{RESUMO}

O objetivo deste experimento foi avaliar o efeito da suplementação de gordura por um curto período de tempo (flushing) sobre o desempenho reprodutivo (aparecimento de estro pós-parto, anestro, intervalo de estro pós-parto, intervalo entre partos, número de inseminações artificiais, intervalo entre as inseminações artificiais e percentagem de prenhez) de fêmeas de corte no pós-parto, submetidas ao desmame precoce. Foram utilizadas 258 vacas, dos grupos genéticos: Aberdeen Angus $(n=21)$, Aberdeen Angus $x$ Canchim $(n=20)$, Canchim $x$ Aberdeen Angus $(n=52)$, Charolês $\times$ Caracu $(n=29)$, Charolês $(n=$ 18), Cachim $(n=34)$, Caracu $(n=35)$, Caracu $x$ Charolês ( $n=26)$ e Purunã $(n=23)$, com peso vivo médio de 418,1 kg e diagnóstico de prenhez positivo. Os animais foram distribuídos nos tratamentos flushing (30\% de grão de soja $+70 \%$ de milho) e não suplementados. Foram avaliados três períodos de acordo com a ordem de parição: setembro, outubro e novembro. Não houve efeito do flushing sobre a percentagem de estro pósparto $(82,9 \%)$, percentagem de anestro $(17,0 \%)$, intervalo de estro pós-parto (100,5 dias), peso ao nascer do bezerro $(34,2 \mathrm{~kg})$, número de inseminações artificiais $(1,4)$, intervalo entre a $1^{\text {a }}$ e a $2^{\mathrm{a}}$ inseminação artificial (19,2 dias), intervalo entre a $2^{\mathrm{a}}$ e $3^{\mathrm{a}}$ inseminação artificial (19,9 dias) e percentagem de prenhez $(54,6 \%)$. O flushing

Recibido: 25-6-07. Aceptado: 17-11-08.

\section{AdDitional KEYWORdS}

Anestrus. Calving interval. Estrus. Lipids.

reduziu o intervalo entre partos (391,1 vs. 399,3 dias). O período de novembro apresentou menor percentagem de estro pós-parto $(70,6 \%)$, percentagem de anestro $(29,4 \%)$, intervalo de estro pós-parto (90,3 dias), intervalo entre partos (378,3 dias), número de inseminações artificiais $(1,2)$, intervalo entre a $1^{\mathrm{a}}$ e a $2^{\mathrm{a}}$ inseminação artificial (11,5 dias) e percentagem de prenhez $(27,5 \%)$.

\section{SUMMARY}

The objective of this experiment was to evaluate the effects of short-time fat supplementation (flushing) on the reproductive performance (postpartum estrus, postpartum anestrus, postpartum estrus interval, calving interval, number of artificial insemination, artificial insemination interval, and pregnancy rate) of postpartum beef cows, submitted to the early weaning. Two hundred and fifty eight beef cows were used from the following genetic groups: Aberdeen Angus $(n=21)$, Aberdeen Angus $\times$ Canchim $(n=20)$, Canchim $x$ Aberdeen Angus $(n=52)$, Charoles $x$ Caracu $(n=29)$, Charolais $(n=18)$, Cachim $(n=34)$, Caracu $(n=35)$, Caracu $x$ Charoles $(n=26)$ an Purunã ( $n=23)$, with $418.1 \mathrm{~kg}$ of body weight and all pregnant. These animals were split into two types of treatments: flushing (30\% soybean full + 
$70 \%$ corn) and no supplementation. There was no flushing effect on postpartum estrus percentage (82.9\%), anestrus percentage (17.0\%), postpartum estrus interval (100.5 days), calf birth weight $(34.2 \mathrm{~kg})$, number of artificial insemination (1.4), interval between the $1^{\text {st }}$ and the $2^{\text {nd }}$ artificial insemination (19.2 days), interval between the $1^{\text {st }}$ and the $2^{\text {nd }}$ artificial insemination (19.9 days), and pregnancy percentage (54.6\%). The flushing reduced calving interval (391.14 vs. 399.31 days). The November period had lower postpartum estrus percentage $(70.6 \%)$, anestrus percentage (29.4\%), postpartum estrus interval (90.3 days), calving interval (378.3 days), number of artificial insemination (1.2), interval between the $1^{\text {st }}$ and the $2^{\text {nd }}$ artificial insemination (11.5 days), and pregnancy percentage $(27.5 \%)$.

\section{INTRODUÇÃO}

As sementes de oleaginosas são capazes de aumentar a densidade energética da dieta e ao mesmo tempo permitir uma alta relação volumoso: concentrado, sem prejudicar o ambiente ruminal, ao contrário do que ocorre com fontes ricas em amido (Coppock e Wilks, 1991). Além disso, a utilização de gordura na dieta pode estar relacionada ao aumento das concentrações sangüíneas de colesterol. O colesterol é o precursor de progesterona e está associado com a manutenção da gestação. Este mecanismo aumenta o tempo de vida de corpo lúteo e, conseqüentemente, a sobrevivência embrionária (Staples et al., 1998).

De Fries et al. (1996), utilizando 40 vacas Brahman suplementadas com dieta controle (3,7\% de extrato etéreo) e com óleo de arroz (5,2\% de extrato etéreo), obtiveram sucesso com a inclusão do óleo. Este aumento no número de folículos possibilitou aumento da taxa de prenhez para os animais que receberam o óleo de arroz (91,5\%) em relação à dieta controle (71,4\%). Da mesma forma, Lammoglia et al. (2000), suplementando 246 novilhas, de três grupos genéticos diferentes (Hereford, Limousin e Peidmontese), com uma dieta baixa (1,9\% extrato etéreo) e uma dieta alta (4,4\% extrato etéreo) em gordura, utilizando a semente de cártamo, observaram que a dieta com $4,4 \%$ de gordura aumentou a percentagem de novilhas púberes no início da estação de monta.

Além da importante manipulação da dieta, outras técnicas de manejo mais antigas têm sido utilizadas para aumentar a produtividade das vacas de corte, como, por exemplo, o desmame precoce (Short et al., 1990; Neville e McCormick, 1981). Esta técnica baseia-se no princípio de que a vaca não apresenta estro fértil logo após a parição devido à amamentação e à presença do bezerro. Várias vezes, essas metodologias apresentam resultados satisfatórios, elevando a taxa de natalidade, quando comparadas com o sistema tradicional de cria do bezerro ao pé da vaca e desmame próximo aos 7 meses de idade.

Todavia, existem poucos trabalhos avaliando o efeito da suplementação de gordura por curto período de tempo (flushing) associado com desmame precoce em vacas de corte no pós-parto sobre o desempenho reprodutivo. Desta forma, o objetivo deste trabalho foi avaliar o efeito do flushing sobre a percentagem de estro pós-parto, percentagem de anestro, intervalo de estro pós-parto, intervalo entre partos, número de inseminações artificiais, intervalo entre as inseminações artificiais e percentagem de prenhez em vacas de corte submetidas ao desmame precoce.

\section{MATERIAL E MÉTODOS}

O experimento foi realizado na Estação Experimental da Fazenda Modelo do Instituto Agronômico do Paraná (IAPAR), localizado no município de Ponta Grossa (PR). Conforme a classificação de Koeppen, o clima da região se enquadra na variedade Cfb, temperado sem estação seca, com temperatura média anual de $17,6^{\circ} \mathrm{C}$, média das máximas de $24,3^{\circ} \mathrm{C}$ e média das mínimas de $8,5^{\circ} \mathrm{C}$. A precipitação média anual é de 1400 $\mathrm{mm}$, sendo as chuvas mais freqüentes no período de primavera - verão e mais escassas no outono. O solo é classificado como

Archivos de zootecnia vol. 59, núm. 225, p. 132. 
Latossolo Vermelho - Escuro de textura média. O período experimental foi realizado a partir de $1^{\circ}$ de setembro de 2001 a 15 de setembro de 2002, de acordo com o período de estação de monta e parição das vacas. Foram utilizadas 258 vacas de corte respectivas aos seguintes grupos genéticos: Aberdeen Angus $(n=21)$, Aberdeen Angus $x$ Canchim $(n=20)$, Canchim x Aberdeen Angus $(n=52)$, Charolês x Caracu $(n=29)$, Charolês $(n=18)$, Cachim $(n=34)$, Caracu $(n=$ 35), Caracu x Charolês ( $n=26)$ e Purunã ( $n=$ 23), com peso vivo médio de 418,1 kg. Todas as fêmeas pariram antes de entrar no experimento. As vacas apresentavam escore corporal médio igual a 3,5, numa escala que varia de 0 a 5 pontos. As vacas estavam entre a segunda e sexta parição. Desta forma, não foi usada fêmeas de primeiro parto.

Foram realizados três períodos de flushing, de acordo com a ordem de parto das vacas do ano anterior. A suplementação de gordura (flushing) foi realizada por um curto período de tempo: 3 a 25 de setembro de 2001 (22 dias); 2 a 22 de outubro de 2001 (20 dias) e 6 a 26 de novembro de 2001 (20 dias). Após o nascimento, de acordo com o período, metade das vacas foi distribuída aleatoriamente em dois tratamentos: flushing e não suplementado, sendo todas submetidas ao desmame precoce, aos 70 dias após parto. As vacas foram distribuídas respeitando-se idade, ordem de parto, peso e grupo genético.

Todos os animais permaneceram durante o período experimental em pastagem de campo nativo da região, sendo os dois piquetes de tamanhos iguais e homogêneos em espécies nativas e disponibilidade de forragem. O aporte de lipídios (flushing) foi fornecido 75 dias após o parto e 15 dias antes da nova estação de monta, respeitando o intervalo para que ocorresse a involução uterina. A ração do flushing foi constituída de $30 \%$ de grão de soja quebrado e $70 \%$ de milho moído, com $8,1 \%$ de extrato etéreo. Os animais, pertencentes ao tratamento do flushing, receberam o concentrado ad libitum, em comedouros dispostos dentro do piquete, para evitar a competição entre os animais.

As fêmeas em estro foram detectadas pelo método visual e uso de rufiões (1 rufião para cada 30 vacas), iniciando 90 dias após o parto anterior. Após a detecção do estro, as vacas foram separadas do lote e foi realizado à inseminação artificial. Todas as vacas, quando retornaram ao cio após a primeira ou segunda inseminação foram submetidas à outra inseminação. Foi avaliado o aparecimento do estro pós-parto, anestro, intervalo entre partos, número de inseminações artificiais realizadas, intervalo entre as inseminações artificiais e percentagem de prenhez. O intervalo do estro pós-parto (ICPP) foi obtido subtraindose a data do primeiro aparecimento de estro pós-parto pela data do parto, transformados em dias individuais para cada vaca. Da mesma forma, o intervalo entre partos (IEP) de cada vaca foi calculado subtraindo-se a data do segundo parto pela data do primeiro parto e assim obtendo-se os intervalos individuais para cada animal.

O desmame precoce foi realizado a partir dos 70 dias após o nascimento dos bezerros. O nascimento dos bezerros ocorreu entre os meses de maio a julho do ano de 2002. Após o desmame, os bezerros foram transferidos para um piquete individual. Nesse local foi fornecida uma dieta concentrada constituída de $25 \%$ de farelo de soja $+71 \%$ de milho moído + 4\% de sal mineral em pastagem de campo nativo.

O controle sanitário do rebanho foi realizado através da vacinação contra carbúnculo sintomático e gangrena gasosa (duas vacinações até o desmame e reforço anual até os dois anos); brucelose nas novilhas com idade em torno de quatro meses; IBR e leptospirose, sendo a primeira dose realizada junto com a vacina contra febre aftosa e o reforço no dia 15 de junho de 2001. Ambas foram repetidas anualmente. A evermifugação foi realizada quatro vezes ao ano, sendo utilizado Ripercol no outono/inverno e Ricobendazole na primavera/verão. 
A análise dos dados foi realizada utilizando o programa Statistical Analysis System (SAS, 1996). O intervalo de aparecimento de estros pós-parto, intervalo entre partos das vacas, intervalo entre as inseminações artificiais foram analisadas pelo método dos quadrados mínimos, utilizando o procedimento General Linear Models (GLM), que considera o desbalanceamento do número de observações(SAS, 1996). Omodelo estatístico utilizado foi o seguinte:

$$
\begin{aligned}
& Y_{i j k l m}=\mu+P_{i}+T_{j}+G_{k}+P \times T_{i j}+T^{\times} G_{j k}+b_{1}\left(I_{i j k l}\right. \\
& -\hat{i})+\mathrm{e}_{\mathrm{ijklm}}
\end{aligned}
$$

onde:

$Y_{\mathrm{i} k \mathrm{klm}}$ é a variável resposta associada ao m-ésima vaca;

$\mu$ a média geral;

$P_{i}$ é o efeito fixo do i-ésimo período;

$T_{j}^{\prime}$ o efeito fixo do j-ésimo tipo de suplementação;

$G_{k} 0$ efeito fixo do k-ésimo grupo genético;

$P \times T_{i j}$ o efeito fixo da interação entre o i-ésimo período e o j-ésimo tipo de suplementação;

$T \times G_{j k}$ o efeito fixo da interação entre o j-ésimo tipo de suplementação e k-ésimo grupo genético;

$b_{1}$, o coeficiente de regressão do termo linear, do peso vivo inicial da vaca, sobre a característica estudada;

$\mathrm{I}_{\mathrm{ijkl}}$ o peso vivo da n-ésima vaca, no início da suplementação;

Î, as médias dos pesos vivos iniciais das vacas a suplementação;

$\left(l_{i j k l}-\hat{I}\right)$, o efeito fixo linear do peso vivo inicial da vaca a suplementação;

$\mathrm{e}_{\mathrm{ijk} \mathrm{k}}$, efeito residual aleatório.

As variáveis que foram expressas em percentagem (percentagem de estro pósparto, anestro e prenhez) das vacas foram analisadas pelo teste do Qui-Quadrado (Gomez e Gomez, 1984), utilizando o procedimento GENMOD do SAS.

\section{RESULTADOSEDISCUSSÃO}

Efeito do flushing: a suplementação das vacas, por um curto período de tempo (15 dias) não alterou ( $p>0,05)$ o número de estro pós-parto, percentagem de estro pós-parto, número de anestro e percentagem de anestro (tabela I).

O intervalo do estro pós-parto, peso ao nascer do bezerro, número de inseminações artificiais por prenhez, intervalo entre $\mathrm{a} 1^{\mathrm{a}} \mathrm{e}$ a $2^{\mathrm{a}}$ inseminação artificial e entre a $2^{\mathrm{a}}$ e $3^{\mathrm{a}}$ inseminação artificial das vacas não foram influenciados ( $\mathrm{p}>0,05)$ pelo uso do flushing (tabela II).

Segundo Williams (1990), a freqüência, a intensidade e a duração da amamentação têm sido consideradas como determinantes primários da duração do anestro pós-parto e, estes fatores foram semelhantes entre os tratamentos por terem número de grupos genéticos semelhantes e terem sido desmamados ao mesmo tempo.

O flushing proporcionou redução $(\mathrm{p}<0,05)$ no intervalo entre partos (tabela II). O intervalo entre partos, segundo Cavalcante et al. (2000) é uma das variáveis mais importantes sobre o índice de fertilidade de um rebanho de corte, pois repercute diretamente na rentabilidade da exploração pecuária limitando a intensidade de seleção, pois o seu prolongamento diminuiu o número de bezerros desmamados e aumenta o intervalo entre gerações.

O menor intervalo entre partos observados nas vacas que receberam o flushing

Tabela I. Efeito do flushing sobre o número de estro pós-parto (NCPP), percentagem de estro pós-parto (PCP), número de anestro (NAN) e percentagem de anestro (PA). (Effect of flushing on number of postpartum estrus (NCPP), percentage of potpartum estrus (\%PCP), number of anestrus (NAN) and percentage of anestrus (\%PA)).

Tratamentos

Flushing Nãosuplementado Média

\begin{tabular}{lccc}
\hline No vacas & 136 & 122 & 129 \\
NCPP & 108 & 106 & 107 \\
PCP & 79,4 & 86,9 & 83,2 \\
NAN & 28 & 16 & 22 \\
PA & 20,6 & 13,1 & 16,9 \\
\hline
\end{tabular}

Archivos de zootecnia vol. 59, núm. 225, p. 134. 
Tabela II. Efeito do flushing sobre o intervalo do estro pós-parto (ICP), intervalo entre partos (IEP), peso ao nascer do bezerro (PN), número de inseminações artificiais (NIA), intervalo entre a $1^{a}$ e a $2^{a} I A(1 I A-2 I A)$ e entre a $2^{a}$ e $3^{a}$ IA (2IA-3IA). (Effect of flushing on postpartum estrus interval (ICP), calving interval (IEP), calf birth weight (PN), number of artificial insemination (NIA), $1^{\text {th }}$ and $2^{\text {th }}$ artificial insemination interval (1IA-2IA) and $2^{\text {th }}$ and $3^{\text {th }}$ artificial insemination interval (2IA-3IA)).

\begin{tabular}{lccc}
\hline & \multicolumn{3}{c}{ Tratamentos } \\
& \multicolumn{3}{c}{ Flushing Não suplementado CV \% } \\
\hline N $^{0}$ vacas & 136 & 122 & - \\
ICP, dias & $100,5 \pm 1,9$ & $102,2 \pm 1,9$ & 17,6 \\
IEP, dias & $391,1 \pm 2,3^{\text {a }}$ & $399,3 \pm 2,6^{\mathrm{b}}$ & 4,64 \\
PN, kg & $34,5 \pm 1,4$ & $34,0 \pm 1,6$ & 32,6 \\
NIA & $1,5 \pm 0,1$ & $1,4 \pm 0,1$ & 38,5 \\
1IA-2IA dias & $17,4 \pm 1,6$ & $19,6 \pm 2,5$ & 46,2 \\
2IA-3IA dias & $20,6 \pm 1,9$ & $19,1 \pm 1,5$ & 13,3 \\
\hline
\end{tabular}

a,b Médias seguidas de letras diferentes, dentro da mesma linha, são diferentes $(p<0,05)$.

(391,1 $\pm 2,3$ dias), em relação às não suplementadas (399,3 \pm 2,6 dias), poderia ser reflexo do efeito da fonte de gordura na alimentação das mesmas. Todavia, o flushing não aumentou o ganho de peso das vacas (Müller, 2003), mas poderia possibilitar o uso da gordura como substância que promoveria o aumento do colesterol sangüíneo sendo este por sua vez o precursor da síntese de progesterona. Delazari et al. (2000), fornecendo grão de soja integral como fonte de lipídios para vacas mestiças Holandês/Zebu no pós-parto (6,3 e 2,5\% de extrato etéreo) obtiveram maiores concentrações de colesterol total e HDL, a partir de quarta semana após o parto, porém não constataram diferença para os níveis de progesterona sérica.

A média do intervalo entre partos observada neste experimento (396,0 dias ou 13,2 meses) foi inferior à média de 431,8 dias ou 14,2 meses observada por Cavalcante et al. (2000), utilizando 405 matrizes Nelores cria- das a pasto no Estado do Maranhão. Da mesma forma, Vargas Jr. et al. (2001) observaram intervalo entre partos de 421,2 dias ou 14,0 meses, em 116 matrizes Nelores puras, no Estado de São Paulo. Todavia, em ambientes semelhantes, Perotto et al. (1994), avaliando fêmeas Nelores e mestiças Guzerá x Nelore, Red Angus x Nelore e Marchiagiana $x$ Nelore, obtiveram valores de IEP acima dos obtidos neste experimento de 17,4 meses. Entretanto, segundo Cavalcante et al. (2000), a média do intervalo entre partos do rebanho brasileiro observado por vários pesquisadores, apresenta grande variação, pois são influenciados por fatores ambientais, genéticos e a interação entre esses dois fatores.

Os dados referentes ao intervalo entre a $1^{\text {a }}$ e $2^{\text {a }}$ IA do tratamento flushing $(17,4 \pm 1,5$ dias) estão próximos dos valores considerados normais para a duração do ciclo estral (18 a 24 dias) (Gonçalves et al., 2000). A duração do ciclo estral do tratamento não suplementado (19,6 \pm 2 ,5 dias), para o intervalo entre a $1^{\text {a }}$ e $2^{\text {a }}$ IA está dentro do normal. Em ambos os tratamentos ocorreram ciclos estrais de duração normal, para o intervalo entre a $2^{\mathrm{a}}$ e $3^{\mathrm{a}}$ IA $(20,6 \pm 1,9$ e 19,1 $\pm 1,5$ dia $)$ para o flushing e não suplementado, respectivamente.

Não houve efeito $(p>0,05)$ do tratamento sobre a percentagem de prenhez, número de estro pós-parto, número de prenhezes por estro pós-parto e percentagem de prenhez por estro pós-parto (tabela III).

A percentagem de prenhez, que engloba todas as vacas do experimento, foi semelhante $(p>0,05)$ entre oflushing e o não suplementado (55,1 vs. 54,1\%). Estes valores são considerados baixos. No entanto, esta percentagem de prenhez significa o mesmo que a percentagem de repetição de cria, uma vez que todas as vacas iniciaram o experimento com prenhez positiva.

A causa principal do alto intervalo entre partos e da baixa repetição de cria é o anestro apresentado pós-parto (Ferreira, 1992; Stagg et al., 1998). Baseadas nestas 
Tabela III. Efeito do flushing sobre a percentagem de prenhez total (PRENT), número de estro pós-parto (NCPP), número de prenhez por estro pós-parto (NPREN/ $C P P)$ e percentagem de prenhez por estro pós-parto (PREN/CPP). (Effect of flushing on total pregnancy rate (PRENT), number of postpartum estrus (NCPP), number of pregnancy to postpartum estrus (NPREN/CPP) and pregnancy rate to postpartum estrus (\%, PREN/CPP)).

\begin{tabular}{|c|c|c|c|c|c|}
\hline & $\mathrm{N}^{0}$ & PREN & VCPP & REN/C & ENN/CP \\
\hline $\mathrm{F}$ & 136 & 55,2 & 108 & 75 & 69,4 \\
\hline NS & 122 & 54,1 & 106 & 66 & 62,3 \\
\hline Média & 129 & 54,7 & 107 & 70,5 & 65,9 \\
\hline
\end{tabular}

F: Flushing; NS: Não suplementado.

constatações foram separadas somente as vacas do experimento que manifestaram estro pós-parto, para as quais foi calculada a percentagem de prenhez somente em relação a estas vacas. Não houve diferença $(\mathrm{p}>0,05)$ entre a percentagem de prenhez e a percentagem de prenhez/estro pós-parto, para os dois tratamentos, constatando-se que outros fatores estão envolvidos na percentagem de prenhez, uma vez as vacas que estavam ciclando apresentaram percentagem de prenhez $(65,9 \%)$ semelhantes, quando se esperava valores maiores por retirar do cálculo os animais em anestro.

Efeito do período: as vacas do período do mês de novembro apresentaram menor $(\mathrm{p}<0,05)$ percentagem de estro pós-parto e maior $(p<0,05)$ percentagem de anestro (tabela IV).

Os resultados sobre a percentagem de estro pós-parto e anestro apresentaram diferenças provavelmente em função do balanço negativo que as vacas apresentaram no terço final da gestação, resultando em maiores exigências quando o campo nativo estava com baixa disponibilidade, debilitando as mesmas. Desta forma, mesmo ganhando peso antes da estação de monta, este acréscimo não foi suficiente para garantir uma atividade ovariana para o surgimento do estro pós-parto, proporcionando maior $(\mathrm{p}<0,05)$ percentagem anestro em relação às vacas do período de setembro e outubro.

De forma similar, houve efeito $(p<0,001)$ do período sobre o intervalo de estro pósparto, intervalo entre partos, número de inseminações artificiais e intervalo entre a primeira e a segunda inseminação artificial (tabela V). Isto demonstra que experimentos realizados em campo nativo são complexos em virtude da variabilidade do mesmo no decorrer do tempo.

Entretanto, ao contrário das outras variáveis, o intervalo entre a segunda e a terceira inseminação artificial não apresentou efeito $(\mathrm{p}>0,05)$ do período, pois no período realizado em novembro ocorreu um número insignificante de três inseminações artificiais/ animal.

O intervalo de estro pós-parto foi menor $(\mathrm{p}<0,05)$ para o período de 6 a 26 de novembro, demonstrando que as vacas deste período manifestaram estro mais rapidamente (27 dias após o desmame precoce) em relação aos demais períodos. $\mathrm{O}$ número de inseminações artificiais foi menor $(\mathrm{p}<0,05)$ para o período de novembro. $\mathrm{O}$

Tabela IV. Efeito do período sobre o número de estros pós-parto (NCPP), percentagem de estro pós-parto (CPP), número de anestro (NAN) e, percentagem de anestro (PA). (Effect of period on number of postpartum estrus (NCPP), percentage of potpartum estrus (CPP), number of anestrus (NAN) and percentage of anestrus (PA)).

\begin{tabular}{lcccc}
\hline & \multicolumn{3}{c}{ Período } & \\
& $3-25 / 09$ & $2-17 / 10$ & $6-26 / 11$ & Média \\
\hline$N^{0}$ vacas & 57 & 99 & 102 & 86 \\
NCPP & 52 & 90 & 72 & 71,3 \\
CPP & $91,2^{\mathrm{a}}$ & $90,9^{\mathrm{a}}$ & $70,6^{\mathrm{b}}$ & 84,2 \\
NAN & 05 & 09 & 30 & 14,7 \\
PA & $8,8^{\mathrm{a}}$ & $9,1^{\mathrm{a}}$ & $29,4^{\mathrm{b}}$ & 15,8
\end{tabular}

${ }^{\mathrm{ab}}$ Médias seguidas de letras diferentes, dentro da mesma linha, são diferentes $(p<0,05)$.

Archivos de zootecnia vol. 59, núm. 225, p. 136. 
Tabela $V$. Efeito do período sobre o intervalo do estro pós-parto (ICPP), intervalo entre partos (IEP), peso ao nascer (PN), número de inseminações artificiais (NIA), intervalo entre a $1^{a}$ e a $2^{a} I A(1 I A-2 I A)$ e entre a $2^{a}$ e $3^{a} I A(2 I A-3 I A)$. (Effect of period on postpartum estrus interval (ICPP), calving interval (IEP), calf birth weight (PN), number of artificial insemination (NIA), $1^{\text {th }}$ and $2^{\text {th }}$ artificial insemination interval (1IA-2IA) and $2^{\text {th }}$ and $3^{\text {th }}$ artificial insemination interval (2IA-3IA)).

\begin{tabular}{lccccc}
\hline & \multicolumn{5}{c}{ Período } \\
& $3-25 / 09$ & $2-17 / 10$ & $6-26 / 11$ & Média & CV \% \\
\hline ICPP, dias & $112,4 \pm 2,8^{\mathrm{b}}$ & $101,2 \pm 2,0^{\mathrm{c}}$ & $90,3 \pm 2,2^{\mathrm{a}}$ & 100,5 & 17,6 \\
IEP, dias & $410,3 \pm 3,0^{\mathrm{c}}$ & $397,1 \pm 2,5^{\mathrm{b}}$ & $378,3 \pm 3,7^{\mathrm{a}}$ & 396,0 & 4,6 \\
PN, kg & $35,6 \pm 1,8$ & $33,7 \pm 1,5$ & $33,6 \pm 2,2$ & 34,2 & 32,6 \\
NIA & $1,6 \pm 0,1^{\mathrm{b}}$ & $1,6 \pm 0,1^{\mathrm{b}}$ & $1,2 \pm 0,1^{\mathrm{a}}$ & 1,4 & 38,5 \\
1IA-2IA, dias & $21,4 \pm 2,1^{\mathrm{b}}$ & $22,6 \pm 1,5^{\mathrm{b}}$ & $11,5 \pm 3,7^{\mathrm{a}}$ & 19,2 & 46,2 \\
2IA-3IA, dias & $20,5 \pm 1,8$ & $19,3 \pm 1,3$ & - & 19,9 & 13,3 \\
\hline
\end{tabular}

${ }^{a b c}$ Médias seguidas de letras diferentes, dentro da mesma linha, são diferentes $(p<0,05)$.

intervalo entre a primeira e a segunda inseminação artificial foi menor $(\mathrm{p}<0,05)$ para o período de novembro (11,5 dias) em relação a outubro e setembro (19,3 e 20,5 dias).

No entanto, este curto intervalo entre a primeira e a segunda inseminação artificial (11,5 dias) poderia indicar a ocorrência somente de manifestação externa de estro, sem ocorrência de estro ovulatório, pois segundo Gonçalves et al. (2000), o ciclo estral, em bovinos, apresenta uma duração de 18 a 24 dias, sendo mais comum 21 dias. Delazari et al. (2000), embora utilizando um plano nutricional alto, com silagem de milho e concentrado à base de grão de soja inte- gral, para vacas mestiças Holandês/Zebu no pós-parto, encontraram ciclos estrais de curta duração após a primeira ovulação, no tratamento grão de soja e na testemunha, de 14,7 a 16,9 dias, respectivamente. O intervalo entre a segunda e a terceira inseminação artificial, para os períodos realizados em setembro e outubro, apresentaram-se dentro da duração normal, com média de 19,9 dias.

Não houve efeito $(p>0,05)$ dentro do período para percentagem de prenhez e percentagem de prenhez por estro pós-parto (tabela VI).

Entretanto, houve diferença $(p<0,05)$ entre os períodos com relação à percentagem

Tabela VI. Efeito do período sobre a percentagem de prenhez total (PRENT), número de estro pós-parto (NCPP), número de prenhez por estro pós-parto (NPREN/CPP), e percentagem de prenhez por estro pós-parto (PREN/CPP). (Effect of period on total pregnancy rate (PRENT), number of postpartum estrus (NCPP), number of pregnancy to postpartum estrus (NPREN/CPP) and pregnancy rate (\%) to postpartum estrus (PREN/CPP)).

\begin{tabular}{lccccc}
\hline Período & Número & PRENT & NCPP & NPREN/CPP & PREN/CPP \\
\hline 03 a 25/09/01 & 57 & $84,2^{\mathrm{a}}$ & 52 & 48 & $92,3^{\mathrm{a}}$ \\
02 a 22/10/01 & 99 & $65,7^{\mathrm{b}}$ & 90 & 65 & $72,2^{\mathrm{b}}$ \\
06 a 26/11/01 & 102 & $27,5^{\mathrm{c}}$ & 72 & 28 & $38,9^{\mathrm{c}}$ \\
Média & 86 & 54,7 & 71,3 & 47 & 65,9 \\
\hline
\end{tabular}

${ }^{a b c}$ Médias seguidas de letras diferentes, dentro da mesma coluna, são diferentes $(p<0,05)$. 
de prenhez e percentagem de prenhez por estro pós-parto. O período de setembro apresentou maior $(\mathrm{p}<0,05)$ percentagem de prenhez (84,2\%) em relação ao mês de outubro $(65,7 \%)$ e este foi superior $(\mathrm{p}<0,05)$ ao mês de novembro (27,5\%). Esta percentagem de prenhez extremamente baixa encontrada no último período de 6 a 26/11/2001 é reflexo da menor percentagem de estro pós-parto (70,6\%), na tabela IV e da maior percentagem de anestro $(29,4 \%)$ associado ao anormal intervalo entre a primeira e a segunda inseminação artificial (11,54 dias), mostrados na tabela V.

O período de setembro apresentou maior $(\mathrm{p}<0,05)$ percentagem de prenhez/estro pósparto, com índices reprodutivos considerados altos (92,3\%) para o sistema extensivo, em campo nativo. Todavia, o mês de outubro apresentou valores médios (72,2\%) diferindo $(\mathrm{p}<0,05)$ do mês de novembro, com índices reprodutivos extremamente baixos (38,9\%). Estes resultados poderiam estar relacionados às restrições severas no terço final da gestação, comprometendo a eficiência

\section{BIBLIOGRAFIA}

Cavalcante, F.A., Filho, R.M., Campello, C.C., Lobo, R.N.B. e Martins, G.A. 2000. Intervalo de partos em rebanho Nelore na Amazônia Oriental. Rev. Bras. Zootecn., 29: 1327-1331.

Coppock, C.E. and Wilks, D.L. 1991. Supplemental fat in high-energy rations for lactating cows: effects on intake, digestion, milk yield, and composition. J. Anim. Sci., 69: 3826-3837.

De Fries, C.A., Neuendorff, D.A. and Randel, R.D. 1996. Fat supplementation influences postpartum reproductive performance in Brahman cows. J. Anim. Sci., 76: 864-870.

Delazari, J.A., Fonseca, F.A., Queiroz, A.C., Pereira, J.C. e Cecon, P.R. 2000. Desempenho reprodutivo, concentrações de progesterona e metabólitos lipídicos no pós-parto de vacas mestiças $H / Z$, submetidas a uma dieta hiperlipidêmica. Rev. Bras. Zootecn., 29: 413-420.

Ferreira, A.M. 1992. Efeito da amamentação na reprodução de vacas: uma revisão. Pesqui. Agropecu. Bras., 27: 27-39. reprodutiva, pois segundo Lobato et al. (2000), uma maior condição corporal ao parto é a primeira das condições para a obtenção de uma nova concepção ao início do próximo período de acasalamento.

\section{CONCLUSÕES}

O flushing de 15 dias pré-estação de cobertura não influenciou a percentagem de estro pós-parto, percentagem de anestro, intervalo do estro pós-parto, peso do bezerro, número de inseminações artificiais, intervalo entre as inseminações e a percentagem de prenhez das vacas submetidas ao desmame precoce. Todavia, a suplementação de gordura por um curto período de tempo (15 dias) reduziu o intervalo entre partos.

A percentagem de estro pós-parto, percentagem de anestro, intervalo do estro pós-parto, intervalo entre partos, número de inseminação artificial, intervalo entre a primeira e a segunda inseminação artificial e percentagem de prenhez foram menores para o período realizado em novembro.

Gomez, K.A. and Gomez, A.A. 1984. Statistical procedures for agricultural research. $2^{\mathrm{a}}$ ed. John Wiley. New York. 680 pp.

Gonçalves, P.B.D., Neves, J.P. e Oliveira, J.F.C. 2000. Fisiologia do ciclo estral. Em: Galina, C. et al. (Ed.) Avanços na reprodução bovina. $1^{\mathrm{a}} \mathrm{ed}$. Ed. Universitária. Pelotas. pp. 11-24.

Lammoglia, M.A., Bellows, R.A., Grings, E.E., Bergman, J.W., Bellows, S.E., Short, R.E., Hallford, D.M. and Randel, R.D. 2000. Effects of dietary fat and sire breed on puberty, weight, and reproductive traits of F1 beef heifers. $J$. Anim. Sci., 78: 2244-2252.

Lobato, J.F.P., Müller, A., Neto, O.A.P. e Osório, E.B. 2000. Efeitos da idade à desmame dos bezerros sobre o desempenho reprodutivo de vacas de corte primíparas. Rev. Bras. Zootecn., 29 (supl1): 2013-2018.

Müller, M. 2003. Fontes de gordura e flushing no desempenho de novilhas e vacas de corte no pós-parto. Tese (Doutorado em Zootecnia).

Archivos de zootecnia vol. 59, núm. 225, p. 138. 
SUPLEMENTAÇÃO DE GORDURA (FLUSHING) EM VACAS DE CORTE NO PÓS-PARTO

Universidade Estadual de Maringá. Maringá. $135 \mathrm{pp}$.

Neville, W.E. and McCormick, W.C. 1981. Performance of early and normal weaned beef cows and their dams. J. Anim. Sci., 52: 715-724.

Perotto, D., José, W.P.K. e Abrahão, J.J.S. 1994. Idade ao primeiro parto e intervalo entre partos de fêmeas bovinas Nelore e de mestiças Guzerá $x$ Nelore, Red Angus $x$ Nelore e Marchigiana $x$ Nelore. Em: Reunião Anual da Sociedade Brasileira de Zootecnia. 31, 1994. Maringá. Anais... Maringá: SBZ. p. 176.

SAS. 1996. Sas/Stat. User's guide. $11^{a}$ ed. SAS Institute. Cary.

Short, R.E., Bellows, R.A., Staigmiller, R.B., Berardinelli, J.G. and Custer, E.E. 1990. Physiological mechanisms controlling anestrus and fertility in post- partum beef cattle. J. Anim. Sci., 68: 799-816.
Stagg, K., Spicer, L.J., Sreenan, J.M., Roche, J.F. and Diskin, M.G. 1998. Effect of calf isolation on follicular wave dynamics, gonadotropin and metabolic hormone changes, and interval to first ovulation in beef cows fed either of two energy levels postpartum. Biol. Reprod., 59: 777-78.

Staples, C.R., Burke, J.M. and Thatcher, W.W. 1998. Symposium: optimizing energy nutrition for reproduction dairy cows. J. Dairy Sci., 81: 856-871.

Vargas Jr., F.M., Ratti Jr., J. e Rocha, G.P. 2001. Fatores que atuam sobre o intervalo entre partos e peso aos 205 dias de um rebanho da raça Nelore. Em: Reunião Anual da Sociedade Brasileira de Zootecnia. 38, Piracicaba. Anais... SBZ. Piracicaba. pp. 444-445.

Williams, G.L. 1990. Suckling as a regulator of postpartum rebreeding in cattle: a review. J. Anim. Sci., 68: 831-852.

Archivos de zootecnia vol. 59, núm. 225, p. 139. 